\title{
La educación inclusiva: una vía para la integración*
}

\section{The Inclusive Education: a Way for Integration}

\author{
Karina Uzcátegui Montes** \\ Universidad Autónoma de Madrid, España \\ - Universidad de Tubinga, Alemania. \\ Belkis Cabrera de los Santos Finalé \\ Universidad Autónoma de Madrid, España \\ - Save the Children, España (ONG).

\section{Paola Lami} \\ Universidad Autónoma de Madrid, España \\ - Fundación Hazloposible, España.
}

Recibido: 24 de octubre de 2011 Revisado: 14 de diciembre de 2011 Aceptado: 10 de enero de 2012

\section{Resumen}

En este artículo se presentan algunos lineamientos teóricos respaldados por prácticas exitosas que aportan una visión alternativa en el diseño de políticas educativas que potencian la construcción de una nueva ciudadanía; lo cual, al mismo tiempo, conlleva a la cohesión social y a la integración de los ciudadanos. Partimos de un análisis crítico de las medidas compensatorias que se llevan a cabo en España en el ámbito educativo y presentamos la educación inclusiva, destacando a las comunidades de aprendizaje como un modelo basado tanto en la igualdad de oportunidades como de resultados, que garantiza tanto el acceso como la calidad en la educación. De esta manera, argumentamos una alternativa para gestionar la diversidad en la escuela, que pone atención, fundamentalmente, en la equidad, procurando que todo el alumnado alcance niveles de excelencia y en la integración, como elementos claves para que los movimientos migratorios potencien el desarrollo humano.

Palabras clave: educación inclusiva, desarrollo humano, comunidad de aprendizaje, movimiento migratorio, aprendizaje dialógico, interculturalidad.

Artículo de investigación. Investigación en el programa del Máster en Inmigración, Refugio y Relaciones Intercomunitarias de la Universidad Autónoma de Madrid, 2009.

** Correspondencia: belkismadrid@hotmail.com, Karina Uscátegui Montes <karinamontes@hotmail.com>, Paola Lami <paolalami@hotmail.com> 


\section{Abstract}

This article presents some theoretical guidelines, supported by best practices, which provide new insight into the design of educational policies that promote the construction of a new citizenship, at the same time this leads to social cohesion and integration of citizens. We started from a critical analysis of the compensatory measures in education that are carried out in Spain and we presented the inclusive education, highlighting the Learning Communities, as a model based on equal opportunities and results, which guarantees the access to education and its quality. We argue thereby an alternative way to manage diversity within the school, which basically focuses on equality, by ensuring that all students reach levels of excellence, and on integration, as key elements to make migratory movements promote human development.

Key words: inclusive education, human development, learning communities, migratory movement, dialogic learning, interculturalism.

\section{Introducción}

Nos encontramos en un mundo globalizado donde la desigualdad de oportunidades crece en todas las sociedades y culturas, y son precisamente esas diferencias las que motivan, en la mayoría de los casos, los movimientos humanos. Es así como en estos tiempos, al hablar de migraciones, se habla necesariamente de derechos, minorías, convivencia con un "otro" diferente. Touraine (1998) señala cómo el reconocimiento del otro no se limita a la relación interpersonal, sino que presupone condiciones institucionales, políticas y jurídicas de la libertad personal y de la comunicación entre sujetos. Escuchamos hablar también de justicia, igualdad, cultura y capacidad, se reorganizan nuevos discursos, teorías, leyes, disposiciones, normativas y visiones, así como se profundizan los debates sobre las consecuencias de este "nuevo ordenamiento" para los pueblos y personas.

Dentro de este amplio abanico de respuestas y miradas múltiples nos identificamos con una visión positiva de la movilidad humana, que la coloca como generadora de beneficios y oportunidades, tanto para las personas que migran y sus familiares, como para las sociedades de origen y destino. En este sentido, compartimos el criterio de que los desplazamientos, tanto nacionales como internacionales, constituyen un potencial para mejorar el desarrollo humano. No obstante es necesario analizar y valorar, qué políticas son más adecuadas para que la migración acarree un saldo positivo en términos de desarrollo humano para todos los grupos y personas implicados en este fenómeno, de manera que se satisfagan las necesidades sociales y a su vez se desarrollen las capacidades, habilidades, libertades e ideas de todas las personas, garantizando en definitiva la integración.

Asumiendo que dicha integración depende del reconocimiento y cumplimiento de los derechos de las personas y que la educación es una dimensión del desarrollo humano, coincidimos con el argumento planteado por el PNUD (2009) en el IDH (2009), donde se enfatiza que una de las políticas claves para alcanzar mejores logros, en el marco del mismo, para los migrantes y las comunidades de destino, es garantizar la igualdad en el acceso y el trato en servicios básicos como la educación. No es suficiente, por tanto, garantizar la posibilidad de una educación para todas las personas (igualdad de oportunidades), es necesario que se ofrezca una educación de calidad, de manera que se asegure el pleno desarrollo personal y la inserción en el mundo del trabajo, es decir, un lugar en la sociedad de la información y la capacidad para participar en una vida activa y ciudadana responsable de cada miembro de la sociedad (igualdad de resultados).

Tomando en cuenta estas premisas, el objetivo de este artículo es argumentar la importancia de un 
modelo educativo de calidad que propicie la integración de los jóvenes inmigrantes y de la sociedad en general. Intentamos de este modo recrear ideas y hacer una sistematización de aportes sustentados en la teoría y en la praxis para el diseño de políticas educativas que fomenten el desarrollo humano.

\section{Un camino que NO conduce a la integración}

Tomando como horizonte la integración, se asume en este apartado una posición crítica respecto a las pistas (leyes, políticas y prácticas) que se han dado como respuesta a la nueva realidad de la escuela actual en España, entendiendo que no conducen a la meta deseada. En este sentido, se analizará lo referente a la escolarización y al fracaso escolar para visualizar lo que ocurre en el sistema educativo español; se presentarán así las medidas compensatorias y estereotipos que se generan al aplicarlas.

Si bien la escolarización en España ha tenido una evolución favorable, no se puede afirmar lo mismo respecto a los índices de fracaso escolar. En este sentido, el Plan Estratégico de Ciudadanía e Integración ( $\mathrm{PECl}, 2007-2010)$ señala que los alumnos inmigrantes están presentado un fracaso escolar que, en muchos casos, alcanza índices superiores a la media nacional. Respecto a las causas de dicho fenómeno, Fernández recalca que "un problema que se esconde bajo el fracaso escolar radica en la coexistencia de dos mundos, uno dominante y otro minoritario y desvalorizado, en los que estos niños y niñas viven inmersos, dos compartimientos estanco perfectamente cerrados y aislados, sin nada que los conecte" (2006). En la misma línea de argumentación, comenta García Castaño "este fenómeno del fracaso escolar no es un problema exclusivo de "los inmigrantes" o de sus hijos e hijas, sino más bien de la situación socioeconómica desigual en la que se encuentran los grupos minoritarios excluídos, entre los que se encuentran algunas poblaciones inmigrantes" (2008).
Para erradicar y evitar las situaciones de fracaso escolar, y en aras de garantizar la igualdad social, se conciben medidas de educación compensatoria, ${ }^{1}$ aunque este tipo de prácticas tienen diferencias entre sí, el criterio que tienen en común es la agrupación homogénea de la diferencia, teniendo en cuenta los niveles de conocimiento, y comportamiento del alumnado. Dichas medidas compensatorias están avaladas por la legislación educativa y el PECI (20072010). ${ }^{2}$ Asimismo, el capítulo II, artículo 80 de la Ley Orgánica de Educación (LOE) refiere: “Las políticas de educación compensatoria reforzarán la acción del sistema educativo de forma que se eviten desigualdades derivadas de factores sociales, económicos, culturales, geográficos, étnicos o de otra índole".

Además de las adaptaciones curriculares que se llevan a cabo en agrupaciones homogéneas, se realizan otras prácticas excluyentes en centros con predominio de colectivos desfavorecidos, como son las escuelas públicas, donde se rebaja el nivel educativo en contraste con el que se establece en centros donde el alumnado, en su mayoría, pertenece a familias con un nivel socioeconómico alto. A ello se refiere Aubert cuando señala:

[...] en las escuelas e institutos de élite, los currículos se orientan a objetivos tales como la competitividad y el esfuerzo. Se crea un ambiente que tiende a preparar a las personas que serán las dirigentes en esta sociedad de la información. Si se propone una asignatura optativa o algún taller, se piensa en un tema que sea importante para competir en la sociedad, como informática e idiomas. En cambio en centros escolares de barrios pobres no

1 Dentro de estas prácticas se destacan las aulas enlace, grupos de compensatoria, programas de garantía social, aulas de compensación externas, centro de educación especial, entre otros.

2 El PECI (2007-2010) menciona que "existen una serie de necesidades sociales y educativas no cubiertas que están limitando el rendimiento y los resultados de los alumnos inmigrantes en las aulas y que requieren ser atendidas por otros servicios de compensación y normalización social. Ante esta situación y para alcanzar el nivel educativo adecuado es necesario prestar una atención prioritaria a las personas que encuentran especiales dificultades como puede ser una parte del alumnado inmigrante, lo cual pasa por desarrollar políticas que prevengan el fracaso escolar, el abandono temprano de los estudios y cualquier otro factor de riesgo que suponga acrecentar las diferencias existentes entre los sectores sociales normalizados y los más desfavorecidos". 
se prioriza ni la competitividad ni el esfuerzo, y a menudo los objetivos son la sociabilidad y la felicidad. La sociabilidad suele asociarse con ser feliz, de esta manera se les prepara para acabar siendo excluídos y excluídas de la sociedad de la información y ser felices con poco sin provocar conflictos [...] (2004).

Se manifiesta en este artículo un claro desacuerdo con todas estas leyes, políticas y prácticas segregadoras que están en detrimento de la integración y que ofrecen como alternativa medidas compensatorias. Tomando el ejemplo de España, vemos como dichas medidas son, en sí mismas, excluyentes y generan situaciones de desigualdad. Compartimos el criterio de Valls (2004) de que estas respuestas al fracaso escolar han llevado al desarrollo de escuelas guetos en las que no se ofrece al alumnado una educación de calidad y es importante aclarar que, en la mayoría de los casos, dichos alumnos pertenecen a grupos sociales vulnerables de familias con dificultades económicas o de grupos sociales minoritarios.

[...] Estas prácticas educativas que pretenden frenar el fracaso escolar parten de una perspectiva más centrada en lo que le falta al alumnado para adecuarse a los cánones que marca la cultura dominante que en sus capacidades y potencialidades. Por esta razón, se llama teorías del déficit a los presupuestos teóricos que sustentan estas perspectivas, que atribuye la falta de éxito escolar de los grupos más desfavorecidos a su falta de interés, su inconciencia e incluso a sus limitaciones intelectuales. Se tiende a identificar de este modo el nivel socioeconómico y educativo de las personas con su grado de motivación o con su procedencia cultural (2004).
Por otra parte, el IDH (2009) ${ }^{3}$ hace referencia a las diferencias existentes en el desempeño escolar entre niños de orígen extranjero, niños migrantes y aquéllos que no tienen antecedentes de migración, declarando que son estos últimos quienes adquieren niveles más elevados de educación, en la mayoría de los casos, y que las medidas homogeneizantes aplicadas en algunos países aumenta la distancia en el rendimiento de estos grupos. Otra reflexión crítica a las medidas compensatorias la sugiere Veredas (2006)

estos dispositivos de compensación actúan como "marcadores" de estos menores ante el resto de los compañeros y minan su autoestima: un niño que ha realizado exitosamente estudios en su país entra en una estructura donde no solo "lo que sabía no vale", sino también donde, en función de esa carencia de saberes apropiados se cuestiona su propia capacidad" (Franzé, 2002).

Aunque la autora solo se refiere a jóvenes inmigrantes, se considera que también se llevan a cabo diferenciaciones entre los hijos de los inmigrantes y aquéllos sin antecedentes de migración, a la hora de valorar las capacidades intelectuales de unos y otros. Tras estas medidas segregadoras existe el supuesto de que habrá una serie de alumnos que no podrán alcanzar los mismos resultados que otros más capaces, dichas expectativas negativas se transmiten e influyen en la autovaloración de la persona como aprendiz y, a su vez, esta percepción de sí mismo determina el rendimiento escolar que reafirma nuevamente la idea de que se es menos capaz.

Tomando en consideración que son los jóvenes inmigrantes y los hijos de personas inmigrantes quienes sufren, en muchas ocasiones, la exclusión

3 “Una buena educación es fundamental para el futuro. Los datos indican que los niños de los migrantes normalmente muestran mejor desempeño que sus padres, pero no alcanzan totalmente el nivel de los niños sin antecedentes de migración, incluso luego de controlar las características socioeconómicas. Sin embargo, hay excepciones, entre ellas Australia y Canadá, donde el desempeño escolar es cercano e incluso excede aquel de los pares originarios. Los países con sistemas de educación que contemplan la temprana división del alumnado en grupos según sus aptitudes, como Alemania y los Países Bajos, son los que muestran las brechas más grandes en rendimiento escolar" (IDH, 2009). 
del espacio normalizado, la percepción que se genera en los alumnos sin precedentes migratorios, respecto a los primeros, puede ser negativa. De esta manera, se construyen y refuerzan estereotipos discriminadores que ponen límites a la convivencia. La formación de estereotipos negativos no solo es generada por las medidas compensatorias, también por el rol de los medios de comunicación en el tratamiento al tema migratorio.

El discurso en los mismos, respecto a la integración escolar de los jóvenes inmigrantes e hijos de inmigrantes, se centra en las problemáticas que genera su presencia en la escuela, como: el fracaso escolar, la segregación en los centros públicos, la concentración de los alumnos extranjeros en las escuelas públicas y el aprendizaje del idio$\mathrm{ma}^{4}$. Este discurso lejos de analizar el fenómeno de la educación y la integración de forma amplia, resalta negativamente la inserción escolar de jóvenes inmigrantes e hijos de inmigrantes en el sistema educativo. Incorpora además un mensaje desalentador y alarmista que construye y refuerza prejuicios y fomenta actitudes racistas y discriminadoras. De este modo, los presenta como una amenaza y como un factor debilitante para la sociedad en general, en lugar de visualizarles como una oportunidad, reflejando sus capacidades creadoras, sus potencialidades y todo lo positivo que aportan al sistema educativo y a la sociedad en general.

Por otro lado, tienden a analizar los problemas que se generan en el aprendizaje dependiendo de la especificidad cultural de los alumnos, soslayando otros elementos como la falta de participación y democratización de la enseñanza, la formación del profesorado, la orientación del aprendizaje y en definitiva la gestión de la educación en general y de los centros en particular; que serían indicadores importantes de medición de la calidad

$4 \quad$ Esto se ejemplifica en la siguiente recopilación de titulares de diarios españoles; en "El Mundo": "Los alumnos inmigrantes repercuten en los índices de fracaso escolar"(Aguilera, 2008); "Alertan de la creación de guetos en los colegios públicos de Inca" (Fueris, 2009); en el diario "El País": "El fracaso escolar y la integración de inmigrantes principales retos de la ESO" (Caballer, 2009). "Las fronteras nacen en la escuela. Una investigación constata que los alumnos españoles e inmigrantes se relacionan poco en el aula y que los de orígen extranjero están menos integrados"(Sevillano, 2008). en la educación en lo que respecta su alcance y su éxito.

Los medios de comunicación deberían contribuir a la creación de representaciones sociales menos estereotipadas y esto depende de la voluntad individual y social. Teniendo en cuenta a (Flecha, 1997) que "son las personas quienes crean los medios, los mensajes y los sentidos de ambos en nuestras existencias". Pero lo cierto es que se construye un imaginario social de homogeneidad cultural que refleja los elementos desfavorables (que hemos visibilizado a lo largo de este apartado) y a la sociedad como un sistema armónico y homogéneo, en la que aparecen actores antagónicos: "nosotros" versus "ellos", identificando a los miembros de determinados colectivos como diferentes y dándoles un trato excluyente.

En una sociedad así, la integración de los individuos toma un carácter bidireccional. Este proceso se refleja, además, en la definición más utilizada de integración, según el PECI (2007-2010)

la integración es más que un estado de cosas en un momento determinado, es un proceso social y dinámico, prolongado en el tiempo, que tiene que ser continuamente reproducido y renovado; en segundo lugar, que la integración requiere un esfuerzo mutuo y bidireccional de adaptación a la nueva realidad, tanto por parte de la población inmigrada, como de la sociedad receptora; y en tercer lugar, que el marco dentro del cual ha de producirse este esfuerzo mutuo está delimitado por los valores básicos de la Unión Europea.

Sin embargo, consideramos que es necesario entender la integración "no solo entre unos (nacionales) y otros (inmigrantes) sino entre todos los individuos que conviven en un determinado territorio y en un determinado momento histórico". (Catep y Sembla, 2008). Por lo tanto "podríamos hablar de que la integración es incluso multidireccional, más que bidireccional. No es un proceso que se dé entre un ellos y un nosotros: hay muchos ellos y muchos nosotros posibles. No hay un nosotros 'normal' y un ellos patológico, que tiene que ser curado de su diferencia que les hace infe- 
riores. Hay, simplemente diferencias, diversidad" (Catep y Sembla, 2008). ¿Cómo descifrar entonces un camino que nos conduzca a la integración en los términos en los que la concebimos?

\section{La escuela inclusiva: un mapa que nos orienta}

El modelo de enseñanza-aprendizaje presente en el diseño curricular español y el andamiaje ideológico y teórico que lo sustenta se está cuestionando de manera sistemática. Concretamente, nos referimos a la crítica de que es objeto la concepción constructivista de la enseñanza-aprendizaje, por su incapacidad de aportar soluciones satisfactorias a los diferentes problemas de los alumnos, entre los que se encuentran principalmente: la falta de motivación, el fracaso escolar, el absentismo o la deserción escolar y la escasa implicación de las familias.

Una nueva forma de entender la práctica educativa se define como modelo de educación inclusiva, que aborda claramente la aceptación de todos, con el derecho inalienable de pertenecer, de no ser excluido. Entendiendo que

la inclusión se opone a cualquier forma de segregación, a cualquier argumento que justifique la separación, a cualquier pretexto en el ejercicio de los derechos a la educación. La inclusión es un planteamiento comprometido que refleja la defensa de unos valores determinados que se relacionan con los fines de la educación, con la forma de sociedad en la que queremos vivir (García Pastor, 1996).

Es así como en la educación inclusiva predominan cuatro ideas que ayudan a explicar su sentido:

la inclusión como un derecho humano; la inclusión como la vía para garantizar la equidad en educación; el derecho humano que tiene cualquier persona a ser educado junto a sus iguales; y por último, la necesidad de que la sociedad asegure el desarrollo de la inclusión (Moriña, 2004).
Finalmente, la educación inclusiva es además una cuestión social que requiere compromiso e implicación de la comunidad en su totalidad. Esto supone que no solo corresponde a las escuelas garantizarla, también la sociedad en su conjunto debe hacerlo. En este sentido hemos de considerar las definiciones de escuela inclusiva definidas por Stainback \& Stainback y Moriña:

Una escuela inclusiva es aquella que educa a todos los estudiantes dentro de un único sistema educativo, proporcionándoles programas educativos apropiados que sean estimulantes y adecuados a sus capacidades y necesidades, además de cualquier apoyo y ayuda que tanto ellos como sus profesores puedan necesitar para tener éxito. Pero una escuela inclusiva va más allá de todo esto, ya que es un lugar al que todos pertenecen, donde todos son aceptados y son apoyados por sus compañeros y por otros miembros de la comunidad escolar para que tengan sus necesidades educativas satisfechas. Uno de los rasgos esenciales de una escuela inclusiva es el sentido cohesivo de comunidad, la aceptación de las diferencias y las respuestas a las necesidades individuales (Stainback \& Stainback, 1999).

Y según Moriña:

El objetivo de la educación inclusiva no es, por tanto, homogeneizar las diferencias, sino el reconocimiento de esas diferencias y la construcción de la escuela como comunidad para que se trabaje con esas diferencias, de forma que cada persona se sienta en algún sentido conectado con lo que se está haciendo en esa aula (2004).

De estos enunciados queremos destacar dos ideas fundamentales que han sido abordadas por diferentes autores como: Booth (1995); Booth \& Ainscow (1997); Barton (2000); y Susinos (2002), entre otros; estas son: incrementar la participación social y reducir los procesos de exclusión que caracterizan al alumnado inmigrante; puntos clave que fomentarían el desarrollo humano. La escuela inclusiva es una pieza fundamental para lograr una sociedad inclusiva; su éxito radica no 
solo en crear una educación de calidad para todos los alumnos, sino en establecer un paso crucial a la hora de ayudar al cambio de actitudes discriminatorias, para crear comunidades de bienvenida y desarrollar una sociedad abierta que incluya a todos y todas como personas que tienen derechos y pueden ofrecer recursos. La escuela inclusiva es por tanto un mapa que nos orienta por el camino correcto hacia la integración.

\section{Las comunidades de aprendizaje como un modelo de escuela inclusiva}

"El pionero en desarrollar la idea fue el Programa de Desarrollo Escolar de la Universidad de Yale (School Development Program), en el año 1968. Años más tarde le sigue la experiencia de las Accelerate Schools (escuelas intensivas aceleradas) en 1986, creadas en Estados Unidos para paliar el fracaso del alumnado" (Castanys \& Planes, 2006). Los orígenes de comunidades de aprendizaje en España, se basan en una línea de investigación desarrollada a lo largo de varios años por el Centro Especial de Investigación en Teorías y Prácticas Superadoras de Desigualdades (CREA) de la Universidad de Barcelona. Según Racionero \& Serradell (2005) dichas comunidades están basadas en teorías y prácticas inclusivas, igualitarias y dialógicas que han mostrado su utilidad al aumentar el aprendizaje y la solidaridad. Estas presentan principios comunes como son: el diálogo igualitario, la dimensión instrumental, el fomento de expectativas positivas, la igualdad de diferencias y la superación del fracaso escolar.

Comunidades de aprendizaje es la propuesta de educación inclusiva que se destaca como un camino necesario no solo para la integración de los jóvenes inmigrantes, sino también de todos aquéllos jóvenes que forman parte del sistema educativo. De esta manera, retomamos el concepto de Jaussi (2006), quien considera que:

supone reestructurar todo, desde el aula hasta la organización del propio centro y su relación con la comunidad, basándose en el diálogo y la diversidad de interacciones, para conseguir que todas las personas adquieran los conocimientos y las habilidades necesarias para disminuir las diferencias sociales, para fomentar la participación y potenciar una relación igualitaria entre todas las personas y culturas. Es un proyecto de centro educativo puesto que el objetivo prioritario es conseguir una sociedad de la información para todos/as y romper las dinámicas negativas que muchas veces conlleva las dificultades por problemas de desigualdad, pobreza o carencia de otro tipo. Y es un proyecto del entorno ya que a pesar de que la transformación se proponga al interior del aula o centro no solo afecta a este sino que también se refiere a toda la comunidad en su relación con el centro y en su relación consigo misma.

Se pretende lograr un acceso al conocimiento para todos y todas y una lucha contra la exclusión social, por tanto se requiere la participación de toda la comunidad que necesita un gran número de voluntarios y colaboradores. De esta manera, se organiza un proyecto educativo sobre la base del diálogo igualitario a través de una organización democrática donde todas las personas llegan a acuerdos sobre los objetivos, las prioridades y las normas que configurarán su escuela.

En comunidades de aprendizaje se apuesta por una transformación que va más allá de la propia persona, incidiendo en la transformación de las relaciones y de su entorno. En este sentido, basados en una experiencia concreta de un centro donde se realizó el proyecto, se recopiló esta información:

Comenzaron a ver que la diversidad trabajada desde el objetivo de la transformación para la igualdad (y no desde la adaptación a la diversidad) mejoraba el rendimiento. Conociéndose más, también comenzaron a ver que la igualdad de género no se ha logrado en ningún lugar y que las mujeres de todas las culturas debemos unirnos para luchar contra las desigualdades de género existentes en todas ellas. También comenzaron a comprender y analizar que el retraso no era culpa de las personas inmigrantes sino de un sistema 
educativo que no se ha transformado para hacer frente a los cambios de la sociedad y de sus alumnas y alumnos (Puigvert \& Santacruz, 2006).

\section{El aprendizaje dialógico: esencia de las comunidades de aprendizaje}

Para Vigotsky, uno de los referentes teóricos del aprendizaje dialógico, los procesos psíquicos, se dan en el marco de las relaciones entre las personas; es decir, primero tienen un carácter interpsicológico y luego intrapsicológico.

Toda función en el desarrollo cultural del niño aparece en acción dos veces, en dos planos: primero en el plano social y después en el psicológico; al principio entre los hombres como categoría interpsíquica y luego en el interior del niño como una categoría intrapsíquica (Vigotsky, 2000).

Según esta concepción la formación de la personalidad del alumno tiene lugar a partir de la interacción con otros, y de la actividad que se produce en los diferentes ámbitos donde transcurre la vida de la persona. El papel de la educación es por tanto determinante para el desarrollo psicológico, en tanto fomente relaciones y un intercambio comunicativo entre todos los agentes y actores sociales.

Es el aprendizaje dialógico que se genera como producto de la interacción y la comunicación con otros, bajo ciertas condiciones que se detallarán a lo largo de este apartado, el que propicia que el alumnado juegue un papel activo en la construcción del conocimiento, se forme en valores y alcance las competencias necesarias para integrarse en la sociedad del conocimiento, por tanto puede decirse que este aprendizaje antecede al desarrollo y lo conduce. La relación entre aprendizaje y desarrollo es clave para Vigotsky por la repercusión que esto tiene en el diagnóstico de capacidades intelectuales y en la elaboración de una teoría pedagógica. Para él, lo que la persona puede hacer con ayuda es, hasta cierto punto, un mejor indicador de su desarrollo mental, de lo que puede hacer por sí sola. Por eso considera necesario determinar como mínimo dos niveles de desarrollo: el de las capacidades reales y el de las posibilidades para aprender con ayuda de los otros. La diferencia entre estos dos niveles es lo que se denomina: zona de desarrollo próximo.

La determinación de esta zona permite caracterizar el desarrollo tomando en consideración no solo las funciones ya maduras o productos finales del desarrollo, sino también aquellas que están en proceso de maduración. Por otra parte, se reconoce la presencia de un otro que ayuda a desarrollar las capacidades y potencialidades del alumno, generando así una expectativa positiva sobre el que aprende, porque lo que hoy puede hacer con ayuda es lo que mañana podrá hacer por sí mismo.

La interacción con un compañero más capaz propicia que el alumno con mayores dificultades pueda utilizar los niveles de ayuda, orientaciones, pistas y pautas que le brinda el otro para arribar a la solución del ejercicio o problema; de modo que va ganando confianza en sí mismo, y se acorta la distancia entre el desarrollo actual y el potencial, de esta forma se evita también la frustración que siente cuando de manera independiente no consigue un objetivo o cuando el otro más capaz lo hace en su lugar. Por otra parte, el más capaz reafirma y consolida su conocimiento al tener que articularlo de modo que sea comprensible para los demás y las dudas que se generan contribuyen a reorganizar sus planteamientos, enriquecerlos o completarlos.

La enseñanza debe organizarse concibiendo estos espacios relacionales y dialógicos que propician el desarrollo de aquello que solo existe como potencial. Espacios de aprendizaje dialógico donde se generan interacciones que tienen lugar en grupos heterogéneos (entre personas que tienen diferentes niveles), que favorecen la atención a las necesidades individuales, ya que tanto el profesor como el grupo son co-responsables de que cada uno obtenga los mejores resultados posibles y es 
así como se promueve el avance o desarrollo de todas las partes implicadas ${ }^{5}$.

Desde esta perspectiva comunicativa, los conocimientos no son entendidos como productos terminados o verdades absolutas y únicas que han de ser transmitidos de quien sabe más a quien sabe menos; por el contrario, se conciben como construcciones inacabadas, por tanto, el saber deviene como resultado de un diálogo igualitario. Es por medio de un estilo de interrelación democrática entre el profesor, la comunidad y el aprendiz, que la participación se convierte en un derecho ejercitado en la discusión de los problemas, en la crítica a la realidad y en la búsqueda de soluciones creadoras. La legitimidad del profesor no está puesta entonces en su capacidad para imponer desde una postura autoritaria, sino en su capacidad para dialogar.

Tal y como se ha descrito, en el marco de este modelo educativo se genera un proceso de ayuda mutua en el que todos salen ganando. A través del diálogo y de la colaboración se obtienen los aprendizajes instrumentales, actitudinales y procedimentales necesarios para la integración, en una sociedad que demanda de manera creciente destrezas cognitivas relacionadas con el manejo de la información, habilidades comunicativas, conocimientos idiomáticos, capacidad de trabajo en equipo, pensamiento crítico, creatividad, flexibilidad, autonomía, participación y solidaridad.

Es así como aprender por medio del diálogo y de la interacción en contextos de heterogeneidad, supone asumir un papel activo que trasciende a la escuela como espacio de socialización, fomenta la consciencia ciudadana y contribuye a la recreación y construcción de un nuevo mundo. Bruner (2004) plantea que

[...] una cultura se está recreando constantemente al ser interpretada y renegociada por sus integrantes. Según esta perspectiva, una

5 “[...] Se consideraba antes que cada niño era capaz de razonar, argumentar, demostrar, buscar razones para alguna idea y que del choque de semejantes reflexiones nacía la discusión. De hecho, sin embargo, las cosas suceden de distinta manera. Las investigaciones demuestran que de las discusiones nace la reflexión [...]" (Vigotsky, 2000). cultura es tanto un foro para negociar y renegociar los significados y explicar la acción como un conjunto de reglas o especificaciones para la acción. [...]. La educación es o debe ser uno de los foros principales para realizar esta función, aunque suele ser vacilante en asumirla. Es este aspecto de foro de cultura lo que da a sus participantes una función en la constante elaboración y reelaboración de esa cultura; una función activa como participantes y no como espectadores actuantes que desempeñan sus papeles canónicos de acuerdo con las reglas cuando se producen los indicios adecuados.

Es precisamente esa capacidad transformadora del individuo la que determina su integración, asumiendo que

el hombre integrado, es el hombre sujeto. La adaptación es así un concepto pasivo, la integración o comunión es un concepto activo. Este aspecto pasivo se revela en el hecho de que el hombre no es capaz de alterar la realidad, por el contrario se altera a sí mismo para adaptarse (Freire, 1997).

Ha quedado de este modo demostrado que, para garantizar el éxito escolar de todos y todas, es necesario transformar el contexto de aprendizaje, en lugar de hacer adaptaciones curriculares que aumentan las brechas entre los alumnos, que reproducen y perpetúan las situaciones de desigualdad y exclusión. "[...] En los sectores desfavorecidos, la adaptación del currículo a un entorno que no se transforma se convierte en un refuerzo educativo a las desigualdades que previamente padece. (Flecha, 1997).

Por tanto, en lugar de excluir a los alumnos "menos aptos" del espacio normalizado, debe incrementarse el capital humano, con más docentes en una misma aula o con profesores que brinden apoyo en determinados momentos, para facilitar así el aprendizaje dialógico en un espacio abierto, participativo y heterogéneo en el que se tomen en consideración razones igualitarias. 


\section{Consideraciones finales}

Reafirmamos la idea argumentada en este artículo de que es fundamental repensar una sociedad que fomente la inclusión, y que no acepte "separaciones" entre sus miembros. Es desde ahí que apostamos por la urgente y objetiva mirada, quizás de vuelta a lo heterogéneo, para salvar las diferencias y reconocer la igualdad de derechos, resultados y oportunidades y una forma participativa de construir ciudadanía. Vemos así que la implementación de políticas públicas en el ámbito educativo que promuevan una educación inclusiva, cuyo pilar es el diálogo que procura la igualdad que respeta las diferencias, es un paso esencial para alcanzar la integración y caminar hacia el desarrollo humano.

Las comunidades de aprendizaje como modelo educativo han demostrado su eficacia en la superación del fracaso escolar y en generar una convivencia armónica y pacífica. En los centros transformados en comunidad se confía en el potencial del alumnado y se busca el desarrollo pleno de sus capacidades mediante las relaciones interpersonales de ayuda mutua. La resolución de conflictos, y la mejora de ciertas problemáticas, se realizan mediante el diálogo, el compromiso, la participación y la solidaridad. Valores que se aprenden simultáneamente con las competencias necesarias para ocupar un lugar en la sociedad de la información.

El conocimiento sobre la realidad de diversas comunidades de aprendizaje nos muestra que se construyen y fomentan nuevas relaciones de interacción entre los individuos y la sociedad en general, empezando por los vínculos entre la familia y la escuela,

cuando se abren los canales de participación en verdaderas condiciones de igualdad orientando la acción de todos los agentes educativos en la dirección de la mejora de la educación para todos los niños y niñas, la implicación de la familia se dispara hacia cuotas insospechadas (Aubert, 2004).
El éxito de este modelo de escuela inclusiva se debe a la participación de todas y todos. Es allí donde la familia y la comunidad (se engloba aquí a todos sus actores, entre ellos, asociaciones y ONG) comparten con el profesorado el rol de formadoras y orientadoras; donde pueden soñar con ilusión la escuela a la que aspiran. Esta posibilidad de crear y recrear otros mundos posibles es un ejercicio cotidiano en las comunidades de aprendizaje.

El enfoque intercultural está presente en este modelo de escuela inclusiva que son las comunidades de aprendizaje. Los principios de igualdad en las diferencias y el fomento de la interacción del enfoque intercultural coinciden con los principios del aprendizaje dialógico. Sin embargo, se considera que una interpretación equívoca del enfoque intercultural, en el ámbito educativo, es la de imponer a toda costa las relaciones entre jóvenes de diferentes culturas, quizá por el miedo a la "guetización". Estamos de acuerdo en que deben favorecerse y propiciarse espacios interactivos, el conocimiento, respeto y reconocimiento de cada individuo y la búsqueda de lo común como un motivo para el encuentro con un otro diferente, pero también debe respetarse la libertad de establecer vínculos o lazos a partir de afinidades personales.

Finalmente, se quiere subrayar que una educación de calidad que tenga como meta la integración debe ser: inclusiva, participativa y democrática, ello supone, en definitiva, reconocer a los jóvenes como sujetos de derechos y concebirles como ciudadanos. Los menores deberían disfrutar también de una plena ciudadanía. La convención internacional sobre los derechos del niño ofrece un marco legislativo apropiado y propicio para profundizar en el debate de la ciudadanía. En dicha convención, en los artículos del 12 al 17, se contemplan derechos a la participación y a la intervención en lo público, que deben considerarse tan prioritarios como el resto. La escuela debe ser garantista, veladora y procuradora de esta plena ciudadanía. 
Según De Lucas (2008), la plena ciudadanía ha de incluir la total igualdad en derechos políticos, sociales y civiles; concibiendo los derechos políticos desde una noción más amplia que implica, entre otros, los derechos a la participación y a la intervención en la vida pública. Los jóvenes residentes y autóctonos deberían disfrutar de esta plena ciudadanía, en la que los derechos políticos no se colocan a la saga de los otros.

No debemos olvidar que la energía y la entereza necesarias para encontrar el camino hacia la integración, están en nosotros mismos, como individuos, como miembros de una sociedad, como padres o madres y como agentes diversos. Este desafío puede lograrse abriendo nuevos horizontes para el diálogo entre la sociedad civil, el gobierno, la academia, para el diálogo de todos los actores de la sociedad.

El problema no es la integración escolar en sí misma. El problema somos nosotros, nuestros propios límites conceptuales, nuestra capacidad para diseñar un mundo diferente, un sistema escolar diferente y no homogéneo, en el que cada cual pueda progresar, junto con otros, en función de sus necesidades particulares, y que pueda adaptarse para satisfacer las necesidades educativas de cada alumno, de la mano de un profesorado que acepte y esté preparado para enfrentarse a la diversidad. El problema es, en definitiva, nuestra fuerza y disposición para transformar la realidad que nos rodea (Echeita, 1994).

\section{Referencias}

Aguilera, M. (2008). Los alumnos inmigrantes repercuten en los índices de fracaso escolar. Recuperado de: http: / /www.elmundo.es/elmundo/2008/04/03/baleares/1207223100. html

Aubert, A. (2004). Dialogar y transformar. Barcelona: editorial pedagogía crítica del siglo XXI.
Barton, L. (2000). Sociedad y multiculturalismo. Educación, desarrollo y diversidad, 2, 37-56.

LOE. (2006). Ley Orgánica de Educación 2/2006. Madrid.

Booth, T. (1995). Mapping inclusion and exclusion: concepts for all? En C. Catherine, A. Dyson \& A. Millward (Ed.). Towards inclusive schools?, (pp. 96-126). London: David Fulton publishers.

Booth, T. \& Ainscow, M. (1998). Making comparisons: drawing conclusions. En: T. Booth \& $M$. Ainscow. (Ed.). From them to us, (pp. 232246). London: Routledge.

Bruner, J. (2004). Realidad mental y mundos posibles. Barcelona: Editorial Gedisa.

Caballer, N. (2009). El fracaso escolar y la integración de inmigrantes principales retos de la Eso. Recuperado de: http://www.elpais.com/ articulo/comunidad/valenciana/fracaso/escolar/integracion/inmigrantes/principales/ retos/elpepiespval/20090916elpval_2/tes

Castanys, M. \& Planes, Ll. (2006). Comunidades de aprendizaje en la escuela primaria. Claves para la innovación educativa, 36, 73-78.

Catep \& Sembla. (2008). Integración y ciudadanía. Guía para aplicar la perspectiva de la gestión de la diversidad a los procesos de integración en el ámbito municipal. Ayuntamiento de Parla. Concejalía de Bienestar Social y Área Social.

De lucas, J. (2008). Ciudadanía y derechos humanos. Nuevos retos en un contexto de globalización. (Material para la IX edición del máster en inmigración, refugio y relaciones intercomunitarias). Universidad Autónoma de Madrid.

Echeita. (1994). A favor de una educación de calidad. Cuadernos de Pedagogía, 228, 66-67. 
Fernández, L. (2006). La dialéctica de la inclusión vs exclusión de l@s inmigrantes: retórica y realidad. Recuperado de: http:// www.2eracion_caboverdiana.pdf

Flecha, R. (1997). Compartiendo palabras. El aprendizaje de las personas adultas a través del diálogo. Barcelona: Editorial Paidós.

Freire, P. (1997). A la sombra de este árbol. Barcelona: Editorial el Roure.

Fueris, E. (2009). Alertan de la creación de guetos en los colegios públicos de Inca. Recuperado de: http://www.elmundo.es/elmundo/2009/03/17/baleares/1237327033.html

García Castaño, F., Rubio, M. \& Ouafaa, B. (2008). Población inmigrante y escuela en España: un balance de investigación. En: J. García Roca. (Ed.). La inmigración en la sociedad española. Una radiografía multidisciplinar, (pp. 403471). Barcelona: Editorial Bellaterra.

García Pastor, C. (1996). La iniciativa para conseguir la reunificación de los sistemas de educación general y especial en Estados Unidos. Siglo cero, 27(2), 15-24.

Jaussi, M. (2006). Comunidades de aprendizaje. En: Claves para la innovación educativa. Transformando la escuela: comunidades de aprendizaje, 36, 29-33.

Moriña, A. (2004). Teoría y práctica de la educación inclusiva. Málaga: Editorial Aljibe.

Programa de las Naciones Unidas para el Desarrollo. (2009). informe sobre desarrollo humano 2009. Superando barreras: movilidad y desarrollo humano. Nueva York: Naciones Unidas.

Puigvert, L. \& Santacruz, I. (2006). La transformación de centros educativos en comunidades de aprendizaje. Calidad para todas y todos. Revista de Educación, 339, 169-176.

Racionero, S. \& Serradell, O. (2005). Antecedentes de las comunidades de aprendizaje. Educar, revista del Departamento de Pedagogía Aplicada de la Universidad Autónoma de Barcelona, 35, 29-40.

PECI. (2007-2010). Plan estratégico de ciudadanía e integración 2007-2010. Madrid: Secretaría de Estado de Inmigración y Emigración. Dirección General de Integración de los Inmigrantes.

Sevillano, E. (2008). Las fronteras nacen en la escuela. Una investigación constata que los alumnos españoles $e$ inmigrantes se relacionan poco en el aula y que los de origen extranjero están menos integrados. Recuperado de: http://www.elpais.com/articulo/educacion/fronteras/nacen/escuela/ elpepuedu/20080407elpepiedu_1/tes

Stainback, S. \& Stainback, W. (1999). Aulas inclusivas. Madrid: Editorial Narcea.

Susinos, T. (2002). Un recorrido por la inclusión educativa española. Investigaciones y experiencias más recientes. Revista de Educación, 327, 49-68.

Touraine, A. (1998). Igualdad y diversidad. Las nuevas tareas de la democracia. México: Fondo de Cultura Económica.

Valls, R. (2004). Educar contra el fracaso y la exclusión. Cuadernos de Pedagogía, 341 monográfico, 58-61.

Veredas, S. (2006). Sistema educativo e inmigración: ¿compensa la escuela? Circunstancia, 10. Recuperado de: http://www.fog.es/circunstancia/numero10/art10.htm\#inicio

Vigotsky, L. (2000). Obras escogidas. Tomo III. (2 ed). Madrid: Editorial Visor Dis. 